\title{
Model of monopolistic competition with heterogeneous labor
}

\author{
Alexander Filatov ${ }^{1,2 *}$, and Yury Sokolovsky ${ }^{2}$ \\ ${ }^{1}$ Far Eastern Federal University. School of Economics and Management, Vladivostok, Russia \\ ${ }^{2}$ Irkutsk State University, Institute of Mathematics, Economics, and Informatics, Irkutsk, Russia
}

\begin{abstract}
The paper presents a tool for modelling monopolistic competition markets, based on Dixit-Stiglitz ideology but taking into account heterogeneity at labor market. We analyse several modifications of a two-sector general equilibrium model. In the basic one with two levels of workers qualification their shares are determined endogenously on the base of comparison between the higher wage of the skilled worker and heterogeneous education costs, also taking into account the labor mobility between the manufacture and agriculture sector. The model is generalized for the case of continuous distribution of labor qualification. The impact of the model parameters (ratio of fixed and variable costs, market size, heterogeneity in productivity, elasticity of substitution, etc.) on the obtained equilibrium prices, quantities, wages, number and size of firms, social welfare is investigated.
\end{abstract}

\section{Introduction}

The majority of markets in the modern globalized economy have a structure of the monopolistic competition [1]. They consist of a large number of firms, producing a single variety of a differentiated product, firms don't interact directly, there are no entry or exit barriers, and each firm enjoys market power due to consumers' love for variety.

The classical Dixit-Stiglitz monopolistic competition model [2] and its application to the international trade made by Krugman [3, 4] could explain some effects arisen during last decades in the world economy. These effects, including bilateral flows of very close substitution goods, remained inexplicable within theories based on comparative advantages in labor [5] and in production factors endowments [6]. Nevertheless, this theory based on the assumptions of homogeneous labor productivity and homogeneous firms can't reveal some more observed in reality stylized facts. Particularly, heterogeneity is necessary for majority of the international trade models, though different researches since Bernard and Jensen [7,8] showed that exporters are usually more productive than non-exporters.

Several economists tried to solve this problem. Melitz [9] used quite complicated constructions based on the initially unknown heterogeneous marginal costs of each firm [10,11], but it makes almost impossible to analytically investigate the impact of elasticity of substitution, market size, degree of labor heterogeneity, shares of agriculture and manufacture sectors on the equilibrium parameters: prices, quantities, wages, and welfare. There are several other approaches. Manasse and Turrini [12] supposed that entrepreneurs vary in their ability, so they hire homogeneous workers on a perfectly competitive labor market, but the workers work better for a better entrepreneur. Bartel, Lichtenberg, and Sicherman $[13,14]$ assumed that there exist two technologies - old and more effective new, workers vary in their skills, and the more skilled workers have absolute advantages in both technologies and comparative advantages in the new one. It was shown that at the same time firms with both technologies can co-exist. Bernard, Eaton, Jensen, Kortum [15] and Yeaple [16] also showed that at the same time firms with different productivity can co-exist. They hire different workers and pay them different wages due to their skills.

Our approach also uses this assumption. We don't pay attention on matching mechanisms - they can be different [17], but provide exogenous firm heterogeneity. In general we propose tool for analysing monopolistic competition markets with heterogeneous labor. The worked out models can describe the economical agents' behavior in several situations. They also help estimate consequences of different mechanisms of labor market regulation, possible effects of industrialization and deindustrialization, migration between regions and sectors of economy, the impact of education, taking into account the economic and spatial heterogeneity.

\section{The tool for market analysis}

Let's consider the economy consists of traditional sector $A$ (usually called "agriculture" in spite of the fact that a wide set of services also have the same structure), and modern sector $M$ (traditionally called "manufacture").

*Corresponding author: alexander.filatov@gmail.com 
Agriculture sector produces homogeneous good with constant returns to scale, and manufacture sector continuum $n$ of horizontally differentiated varieties indexed by $i \in[0 ; n]$, with increasing returns to scale. $L$ consumers have identical Kobb-Douglas high level utility functions

$$
u=M^{\mu} A^{1-\mu} \rightarrow \max
$$

It means, that they spend shares $\mu \in(0 ; 1)$ and $(1-\mu)$ of income for manufacture and agriculture good respectively: The low level utility function is CES:

$$
M=\left(\int_{0}^{n}(q(i))^{(\sigma-1) / \sigma} d i\right)^{\sigma /(\sigma-1)} .
$$

When elasticity of substitution $\sigma \rightarrow 1$, we have the low level Kobb-Douglas function also, the modifications of manufacture good are independent; consumers spend the fixed sum of money for each of them. When $\sigma \rightarrow+\infty$, we have the linear utility function describing the perfect substitution goods.

Let denote the total income of all identical consumers by $I$. So under the prices $p(i)$ the $i$-modification demand $q(i)$ can be found as a solution of a problem

$$
\int_{0}^{n}(q(i))^{(\sigma-1) / \sigma} d i \rightarrow \max , \quad \int_{0}^{n} p(i) q(i) d i=\mu I .
$$

Hence we can obtain the very important formula

$$
\frac{q(i)}{q(j)}=\left(\frac{p(j)}{p(i)}\right)^{\sigma} \text {. }
$$

The only production factor is heterogeneous labor. Let the labor demand be

$$
l(i)=f+c(i) q(i)
$$

Here value $c(i)$ means the number of workers needed for one unit production at $i$-firm. If the wage is equal to $w(i)$, the total costs of $i$-firm will be

$$
T C(i)=w(i) l(i) .
$$

Each firm maximizes its own profit:

$$
\begin{aligned}
& \pi(i)=p(i) q(i)-w(i)(f+c(i) q(i))= \\
= & q(i)(p(i)-w(i) c(i))-w(i) f \rightarrow \max _{p(i)} .
\end{aligned}
$$

Solving this problem we can find the optimal price for $i$ modification:

$$
p(i)=\frac{\sigma}{\sigma-1} w(i) c(i)
$$

In the long run the profit of all firms should be zero. Let's find the optimal quantity

$$
q(i)=\frac{f(\sigma-1)}{c(i)}
$$

Using the ratio of prices and quantities we can find the relation between wages and productivities

$$
\frac{w(i)}{w(j)}=\left(\frac{c(j)}{c(i)}\right)^{\frac{\sigma-1}{\sigma}} .
$$

The firm size despite heterogeneity is equal for all firms:

$$
l(i)=f+c(i) q(i)=f+f(\sigma-1)=f \sigma .
$$

\section{The basic model}

The proposed tool helps us to analyse monopolistic competition markets under some additional assumptions. In the basic model let population $L$ consist of skilled manufacture workers (with share $\alpha_{1}$, and productivity $c_{1}=c$ ), unskilled manufacture workers (with share $\alpha_{2}$, and productivity $c_{1}=\gamma c$ ) and farmers (with share $1-\alpha_{1}-\alpha_{2}$ ). Let also agriculture sector be numerator with productivity, wage, and price equal to one, and fixed costs equal to zero: $f_{A}=0, c_{A}=1, p_{A}=1, w_{A}=1$ Then we can obtain the following formulas:

$$
\begin{gathered}
p_{1}=\frac{\sigma}{\sigma-1} w_{1} c, \quad p_{2}=\gamma \frac{\sigma}{\sigma-1} w_{2} c, \\
q_{1}=f(\sigma-1) / c, \quad q_{2}=f(\sigma-1) / \gamma c, \\
w_{1}=\gamma^{(\sigma-1) / \sigma} w, \quad w_{2}=w, \quad l_{1}=l_{2}=f \sigma, \\
n_{1}=\alpha_{1} L / f \sigma, \quad n_{2}=\alpha_{2} L / f \sigma, \quad \pi_{1}=\pi_{2}=0, \\
T R_{1}=T C_{1}=\gamma^{(\sigma-1) / \sigma} f \sigma w, \quad T R_{2}=T C_{2}=f \sigma w, \\
w_{1}=k w=\frac{\mu}{1-\mu} \frac{1-\alpha_{1}-\alpha_{2}}{\alpha_{1}+\alpha_{2} / k_{1}}, \quad w_{2}=w=\frac{\mu}{1-\mu} \frac{1-\alpha_{1}-\alpha_{2}}{\alpha_{1} k_{1}+\alpha_{2}} .
\end{gathered}
$$

In the table 1 we show, how the model parameters $f$, $c, L, \gamma, \sigma, \alpha_{1}, \alpha_{2}, \mu$ impact the equilibrium prices, quantities, revenues, costs, wages, number and size of firms in effective and ineffective sector. Let's denote dependences in the form of primal and inverse proportionality with pluses and minuses, increased and decreased dependences of general form with up and down arrows, independent pairs with points.

Table 1. The impact of model parameters on the equilibrium.

\begin{tabular}{|c|c|c|c|c|c|c|c|c|c|c|c|}
\hline & $p_{1}$ & $p_{2}$ & $q_{1}$ & $q_{2}$ & $T R_{1}$ & $T_{R_{2}}$ & $w_{1}$ & $w_{2}$ & $n_{1}$ & $n_{2}$ & $l$ \\
\hline$f$ & $\bullet$ & $\bullet$ & + & + & + & + & $\bullet$ & $\bullet$ & - & - & + \\
\hline $\boldsymbol{c}$ & + & + & - & - & $\bullet$ & $\bullet$ & $\bullet$ & $\bullet$ & $\bullet$ & $\bullet$ & $\bullet$ \\
\hline $\boldsymbol{L}$ & $\bullet$ & $\bullet$ & $\bullet$ & $\bullet$ & $\bullet$ & $\bullet$ & $\bullet$ & $\bullet$ & + & + & $\bullet$ \\
\hline$\gamma$ & $\uparrow$ & $\uparrow$ & $\bullet$ & - & $\uparrow$ & $\downarrow$ & $\uparrow$ & $\downarrow$ & $\bullet$ & $\bullet$ & $\bullet$ \\
\hline$\sigma$ & $\downarrow$ & $\downarrow$ & $\uparrow$ & $\uparrow$ & $\uparrow$ & $\downarrow \uparrow$ & $\uparrow$ & $\downarrow$ & - & - & + \\
\hline$\alpha_{1}$ & $\downarrow$ & $\downarrow$ & $\bullet$ & $\bullet$ & $\downarrow$ & $\downarrow$ & $\downarrow$ & $\downarrow$ & + & $\bullet$ & $\bullet$ \\
\hline$\alpha_{2}$ & $\downarrow$ & $\downarrow$ & $\bullet$ & $\bullet$ & $\downarrow$ & $\downarrow$ & $\downarrow$ & $\downarrow$ & $\bullet$ & + & $\bullet$ \\
\hline $\boldsymbol{\mu}$ & $\uparrow$ & $\uparrow$ & $\bullet$ & $\bullet$ & $\uparrow$ & $\uparrow$ & $\uparrow$ & $\uparrow$ & $\bullet$ & $\bullet$ & $\bullet$ \\
\hline
\end{tabular}

The institutional restrictions on labor market are also can be taken into account. Particularly the model with fixed wages has been worked out and explored [18]. The 
restrictions can be caused, for example, by government policy (uniform scale of wages, etc) or asymmetric information when transactions between principals (firms) and agents (workers) take place. The mechanisms of effective workers stimulation, included firm profit reallocation, were investigated. The obtained equilibrium was compared with equilibrium in the basic model. The possibility for firms to use different production factors was considered by Echazu and Lugovskyy [19], but it was supposed that the production factors endowments are unlimited.

\section{The model with education}

In both modifications it was supposed that the sector shares are set exogenously. Nevertheless the wage level in industry and agriculture can make workers change the sector. If wages in agriculture are higher than in industry the de-industrialization is quite possible, and in situation of higher wages in industry we can expect the mass migration from countryside to cities. Also we can include into the model the possibility to improve professional skills (at some costs depending on abilities) to get higher wages. Let's formalize these assumptions.

1. The workers can move for free from agriculture to unskilled manufacture sector.

2. The manufacture workers can improve skills under some education costs that depend on $\theta \in[0 ; 1]$ - the uniformly distributed individual "education disability" (see fig.1).

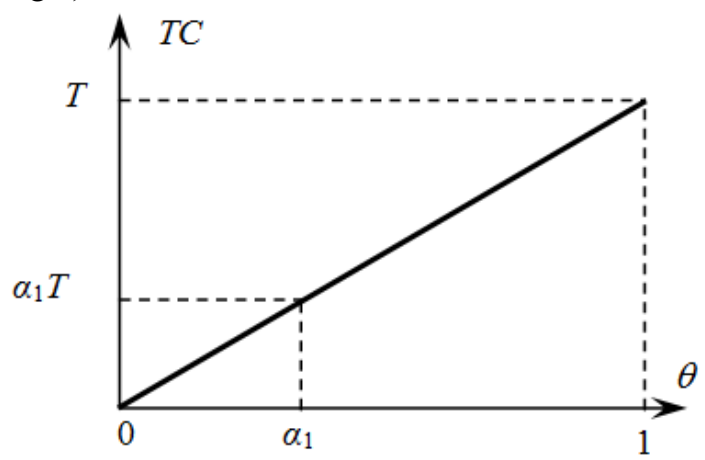

Fig. 1. The education costs.

3. The education costs for the worker with the best ability $(\theta=0)$ is equal to zero, and for the most "alternatively gifted" one $(\theta=1)$ is equal to $T$. So share $\alpha_{1}$ of the most gifted workers with educational disability $\theta \in\left[0 ; \alpha_{1}\right]$ will study.

The equilibrium wages in agriculture and unskilled manufacture sector are the same and equal to one. The workers with low education costs (less than the wages gap) move to the effective sector. Their share can be found from the equality $k_{1} w_{2}-w_{2}=\alpha_{1} T$. Though the equilibrium wage in unskilled manufacture sector is equal to one, we obtain equality $k_{1}-1=\alpha_{1} T$. So the skilled workers share is equal to

$$
\alpha_{1}=\left(k_{1}-1\right) / T \text {. }
$$

From the obtained above formulas it follows

$$
w_{2}=\frac{\mu}{1-\mu} \frac{1-\alpha_{1}-\alpha_{2}}{\alpha_{1} k_{1}+\alpha_{2}} .
$$

We can find also the share $\alpha_{2}$ of unskilled workers:

$$
\alpha_{2}=\mu\left(1-\alpha_{1}\right)-\alpha_{1} k_{1}(1-\mu) \text {. }
$$

If we substitute $\alpha_{1}$ by the obtained above expression we will obtain

$$
\alpha_{2}=\mu-\frac{\left(k_{1}-1\right)\left(\mu+k_{1}(1-\mu)\right)}{T} .
$$

The share of "peasants" can be obtained as $\alpha_{3}=1-\alpha_{1}-\alpha_{2}$ :

$$
\alpha_{3}=(1-\mu)\left(1+\frac{\left(k_{1}-1\right)^{2}}{T}\right) .
$$

Let's estimate how the impact of model parameters has been changed comparing with the basic model. Pluses, minuses, arrows, and points in the table 2 mean the same. Let's also append the line for education costs $T$ and three columns for endogenous shares $\alpha_{1}, \alpha_{2}, \alpha_{3}$ of each sector. The grey cells - are the ones where we can see the changed form of dependence.

The key new results of the model with education are the following:

1. The increasing heterogeneity of industry leads to the higher wages in the effective sector and to increasing qualification of workers via education.

2. The increasing independence of goods leads to the lower wages in the effective sector and decreasing workers studying.

3. The increasing demand on industrial goods doesn't make higher prices and wages.

Table 2. The impact of the model with education parameters on the equilibrium.

\begin{tabular}{|c|c|c|c|c|c|c|c|c|c|c|c|c|c|}
\hline & $p_{1}$ & $p_{2}$ & $q_{1}$ & $q_{2}$ & $T R_{1}$ & $T R_{2}$ & $w_{1}$ & $n_{1}$ & $n_{2}$ & $l$ & $\alpha_{1}$ & $\alpha_{2}$ & $\alpha_{3}$ \\
\hline$f$ & $\bullet$ & $\bullet$ & + & + & + & + & $\bullet$ & - & - & + & $\bullet$ & $\bullet$ & $\bullet$ \\
\hline$c$ & + & + & - & - & $\bullet$ & $\bullet$ & $\bullet$ & $\bullet$ & $\bullet$ & $\bullet$ & $\bullet$ & $\bullet$ & $\bullet$ \\
\hline$L$ & $\bullet$ & $\bullet$ & $\bullet$ & $\bullet$ & $\bullet$ & $\bullet$ & $\bullet$ & + & + & $\bullet$ & $\bullet$ & $\bullet$ & $\bullet$ \\
\hline $\boldsymbol{\gamma}$ & $\uparrow$ & + & $\bullet$ & - & $\uparrow$ & $\bullet$ & $\uparrow$ & $\uparrow$ & $\downarrow$ & $\bullet$ & $\uparrow$ & $\downarrow$ & $\uparrow$ \\
\hline$\sigma$ & $\uparrow \downarrow$ & $\downarrow$ & $\uparrow$ & $\uparrow$ & $\uparrow$ & + & $\uparrow$ & $\uparrow \downarrow$ & $\downarrow$ & + & $\uparrow$ & $\downarrow$ & $\uparrow$ \\
\hline $\boldsymbol{T}$ & $\bullet$ & $\bullet$ & $\bullet$ & $\bullet$ & $\bullet$ & $\bullet$ & $\bullet$ & - & $\uparrow$ & $\bullet$ & - & $\uparrow$ & $\downarrow$ \\
\hline $\boldsymbol{\mu}$ & $\bullet$ & $\bullet$ & $\bullet$ & $\bullet$ & $\bullet$ & $\bullet$ & $\bullet$ & $\bullet$ & $\uparrow$ & $\bullet$ & $\bullet$ & $\uparrow$ & $\downarrow$ \\
\hline
\end{tabular}

\section{The generalized model}

The constructed above basic model has a lot of modifications and generalizations. Particularly it's possible to investigate the situation with any numbers of productivity classes include continuous distribution of workers qualification.

Let the number of workers essential for the unit production at $i$-firm depends on the best worker productivity $c$ and expressed by the following formula:

$$
c(i)=\gamma(i) c, \gamma(i) \in[1 ; \bar{\gamma}] .
$$


Hence the wage level of $i$-firm worker is connected with the best worker wage $w$ and equal to

$$
w(i)=(\gamma(i))^{-(\sigma-1) / \sigma} w .
$$

In the simplest case of uniform distribution the worker qualification can be calculated as follows:

$$
\gamma(i)=1+\frac{i}{n}(\bar{\gamma}-1)
$$

and the prices, quantities, firm size, number of firms are obtained using the formulas

$$
\begin{gathered}
p(i)=\frac{\sigma}{\sigma-1} w(i) c(i), \quad q(i)=\frac{f(\sigma-1)}{c(i)}, \\
l(i)=f \sigma, \quad n=\alpha L / f \sigma
\end{gathered}
$$

The total wage of industrial sector workers is expressed by the integral

$$
\begin{gathered}
W=\int_{0}^{n} l(i) w(i) d i=\frac{\alpha L}{n} w \int_{0}^{n}\left(1+\frac{i}{n}(\bar{\gamma}-1)\right)^{-(\sigma-1) / \sigma} d i, \\
W=\alpha L w \sigma \frac{\bar{\gamma}^{1 / \sigma}-1}{\bar{\gamma}-1} .
\end{gathered}
$$

From the supply and demand balance in agriculture we can find the equilibrium wage level:

$$
\begin{gathered}
(1-\alpha) L=(1-\mu)\left(\alpha L w \sigma \frac{\bar{\gamma}^{1 / \sigma}-1}{\gamma-1}+(1-\alpha) L\right), \\
w=\frac{\mu}{1-\mu} \frac{1-\alpha}{\alpha \sigma} \frac{\bar{\gamma}-1}{\bar{\gamma}^{1 / \sigma}-1} .
\end{gathered}
$$

\section{The Generalized Model with Education}

The proposed in the previous section generalized model was used for more generalized analysis of education and migration [20]. Let's make the following assumptions:

1. Education has multistep structure - from initial level to the best universities.

2. The education of high quality increases labor productivity, educational attainment is connected with costs of production by decreasing linear dependence (fig.2).

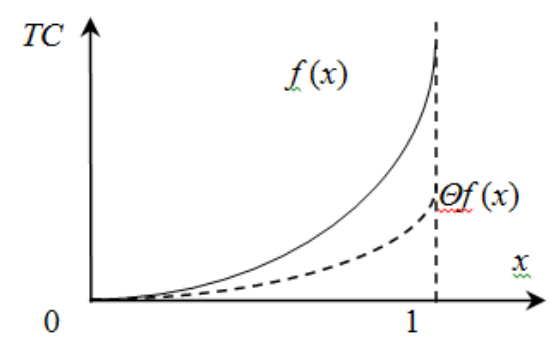

Fig. 2. Educational attainment and costs of education.
3. The education is charged, educational costs are proportional to the "educational skills" $\theta$ and they are given by power function $f(x)=T x^{n}$, where $x$ is a parameter of educational attainment which is between 0 (with no education) and 1 (the maximum of development) (fig.3).

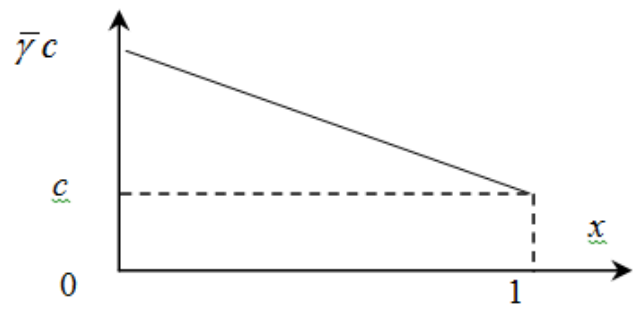

Fig. 2. Educational attainment and productivity of labor.

So, we assume that every additional level of education requires higher increasing of cost incurred and we determine the function of total educational costs as $\theta f(x)$ for taking into account "educational skills".

In this model every person chooses an optimal educational level according to his own skills by focusing on the maximal difference between additional benefits, which are gained after moving to the category of more qualified workers with a higher wage, and costs incurred during the education. Profit can be presented as a financial premium which worker receives having finished study successfully and moved to the necessary sector. Thus, it can be presented in the following way:

$$
F=w(c(x))-w(0)-\theta f(x)
$$

which depends on $x(\theta)$.

It has been possible to prove that when costs of education are linear, the results will be equal to the ones received in the basic model. The most gifted students receive elite education and move to the class of highskilled labor. Untalented ones refuse to obtain education completely and remain in the class on non-skilled workers. Intermediate level of education is not demanded that can be demonstrated by a numerical example with $\rho=3 / 4, n=1, T=10, \bar{\gamma}=8$.

Relations between additional incomes which has been gained as a result of training for people with low, below the average, average, and high inclination to education are denoted by letters $a, b, c, d$. It is obvious that the more worker with low ability studies the bigger his losses are. It is explained by high costs of education which cannot cover estimated profit of higher wage. So, he prefers not to enter the educational establishment (fig.4a). 


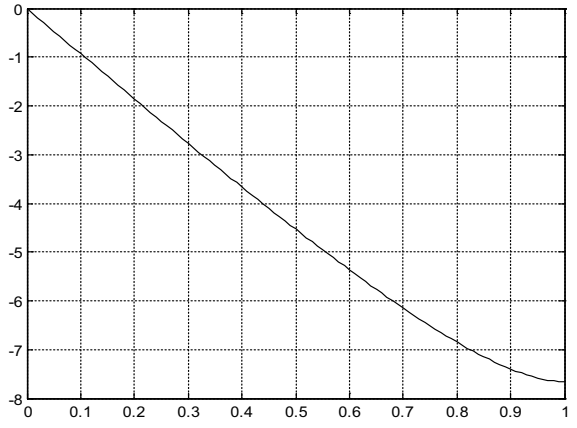

Fig. 4a. Example for "alternatively gifted" person, $\theta=1, x^{*}=0$.

In case of worker with under-average ability at the beginning of education there are only losses as well but after a critical point profit trend becomes positive but final profit is not enough to cover cost incurred (fig. $4 b$ ). As a result of this process worker makes a decision on refusing to be a student and chooses $x^{*}=0$.

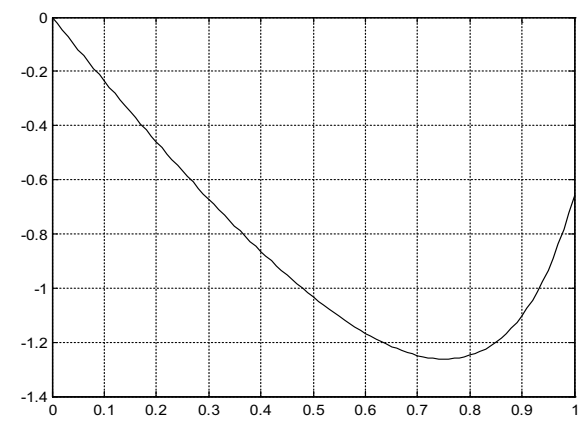

Fig. 4b. Example for medium person, $\theta=0,3, x^{*}=0$.

If we concern the gifted worker it can be observed that his function of profit increases due to the higher quality of education. So, his benefits are maximal at the point $x^{*}=0$ (fig. $4 d$ ). Figure $4 c$ illustrates an intermediate case of profound indifference between these extremes.

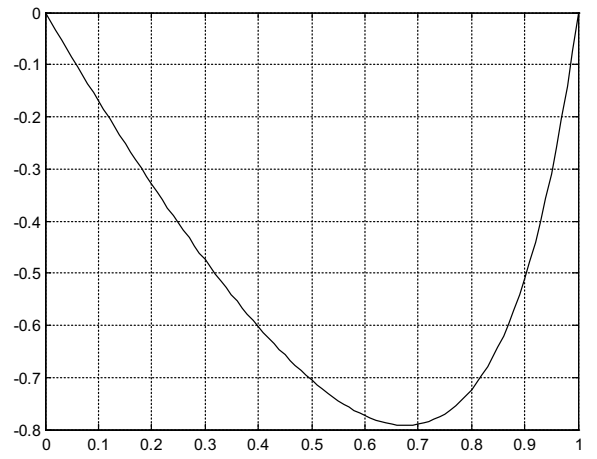

Fig. 4c. Example for talented person, $\theta=0,234, x^{*}=0$ or $x^{*}=1$.

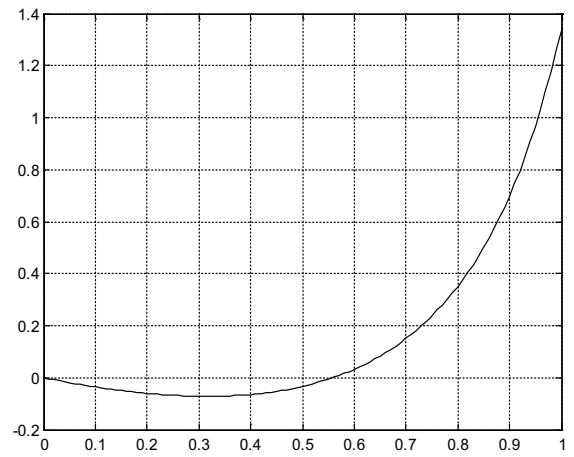

Fig. 4d. Example for genius, $\theta=0,1, x^{*}=1$.

There is no reason to obtain an education for the first two students with lower skills while the most gifted one should seek for studies at the best expensive universities. As far as we can see, there is no any interim decision in case of linear costs: worker either obtains the best education or continues working without studying.

Other parameter points of educational cost function result in different conclusions. For instance, intermediate level standard of education may be optimal in case of quadratic function. Moreover, some minimal education is needed by everybody. We demonstrate results for numerical example with $\rho=1 / 2, n=2, T=10, \bar{\gamma}=8$. Just the same graphics (fig.5) are denoted by letters $a, b$, $c, d$ corresponding with different level of talent for education.

The impact of other model parameters (ratio between fixed and variable costs, economical extent, and elasticity of substitution and so on) on received equilibrium has been also investigated.

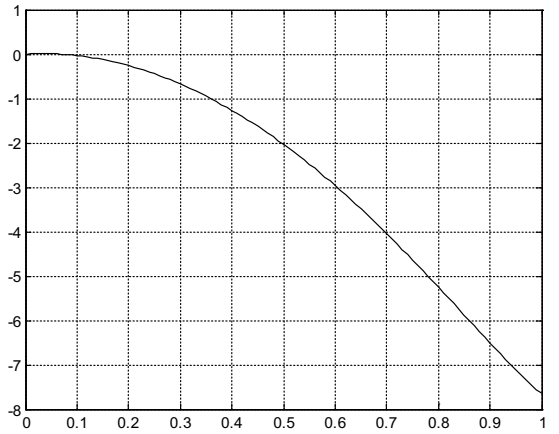

Fig. 5a. Example for "alternatively gifted", $\theta=1, x^{*}=0$.

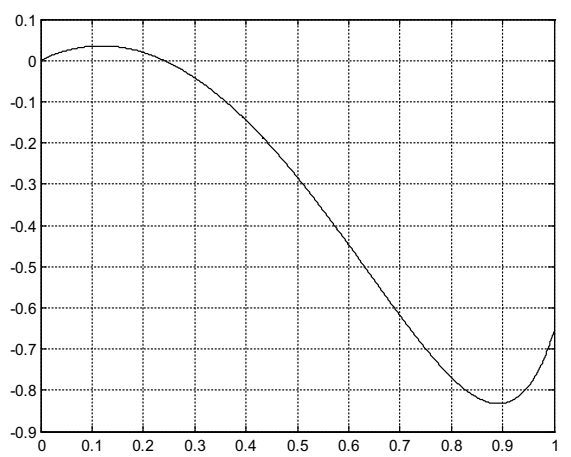

Fig. 5b. Example for medium person, $\theta=0,3, x^{*}=0,1$. 


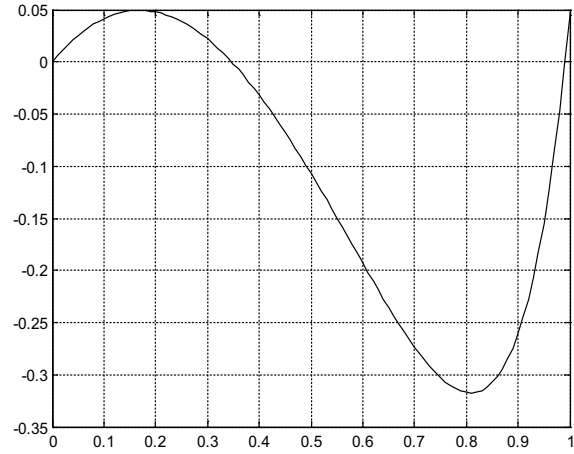

Fig. 5c. Example for talented person, $\theta=0,229, x^{*}=0,15$ or $x^{*}=1$.

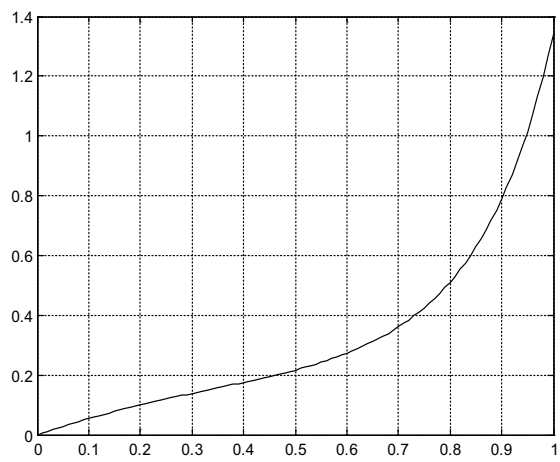

Fig. 5d. Example for genius, $\theta=0,1, x^{*}=1$.

\section{The International Trade Model}

The constructed tools for market analysis (especially generalized model from the section 5) can be also applied to the other fields of economics, particularly to the theory of international or interregional trade. For example, let's investigate the simplest situation of two countries. It's interesting to compare autarky (caused by protectionism tariff police or economic sanctions) with the case of free trade, zero tariffs, and zero transportation costs. Let the workers qualification be distributed as it's shown on the fig.6-7. The first picture describes the distribution of workers' qualification before integration, and the second one in the situation of the joint market of two countries - developed with lower marginal costs and developing with higher ones:

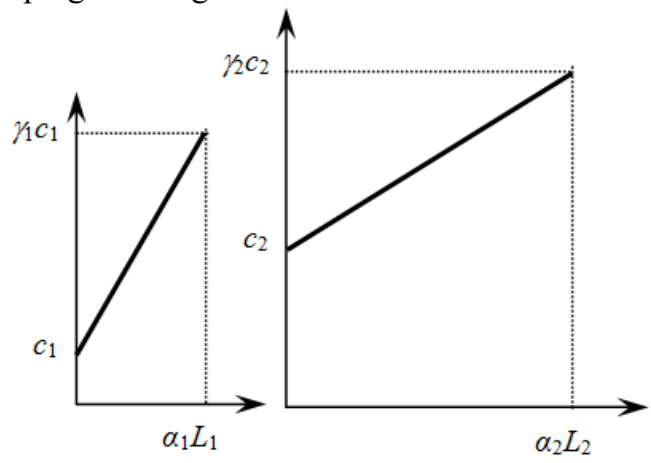

Fig.6. The distribution of workers' qualification before integration.

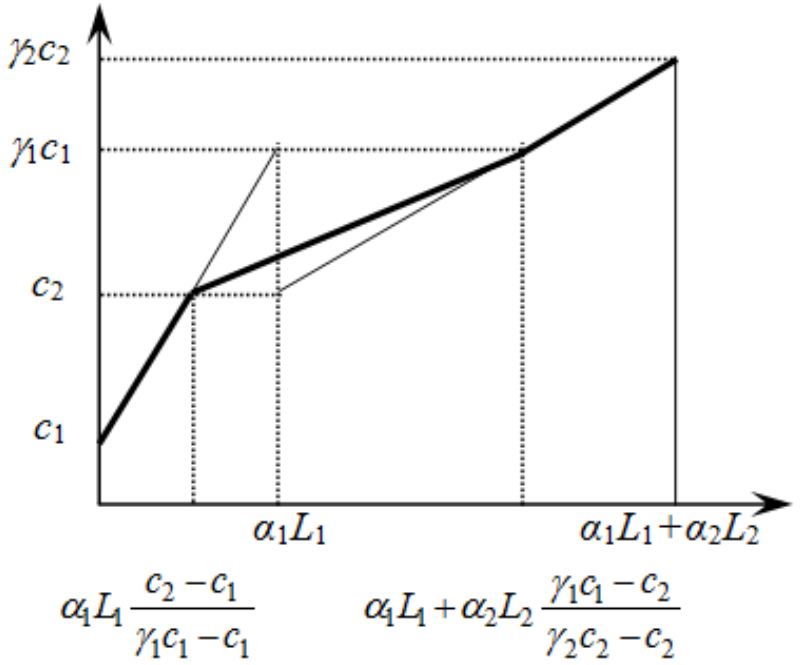

Fig.7. The distribution of workers' qualification after integration.

Using this presented above model it's possible to test some hypotheses. Particularly we showed in [21] that opening borders always expands the variety of goods, increases welfare, converge prices, but sometimes leads to de-industrialization in the country with less effective manufacture sector. It also can be explored the model with transportation cost and tariffs. It's possible to compare equilibriums, obtained in the models with specific and ad valorem tariffs, and give appropriate recommendations.

\section{Conclusions}

The presented tool due to its simplicity and possibility to obtain non-trivial analytical results can be used for solution different problems and giving policy advices for both firms and market regulator. It's also possible to use it for empirical analysis of the data described monopolistic competition markets of different goods in different regions and countries, making forecasts of such markets dynamics, and comparing different mechanisms of regulation.

This research is supported by the RFBR grant № 16-06-00071, and the European Union's Seventh Framework Program FP7/2007-2013/ under REA grant agreement number 609642.

\section{References}

1. E. Chamberlin, The Theory of Monopolistic Competition (Harv. Univ. Press, Cambridge, 1933)

2. A. Dixit, J. Stiglitz, AER, 67, 297-308 (1977)

3. P. Krugman, J. Int. Econ., 9, 469-479 (1979)

4. P. Krugman, AER, 70, 950-959 (1980)

5. D. Ricardo, On the principles of political economy and taxation (John Murray, London, 1817)

6. B. Ohlin, Interregional and International Trade (Harv. Univ. Press, Cambridge, 1968)

7. A. Bernard, B. Jensen, J. Int. Econ., 42, 3-31 (1997) 
8. A. Bernard, B. Jensen, J. Int. Econ., 47, 1-25 (1999)

9. M. Melitz, Econometrica, 71, 1695-1725 (2003)

10. H. Hopenhayn, Econometrica, 60, 1127-1150 (1992)

11. B. Jovanovic, Econometrica, 50, 649-670 (1982)

12. P. Manasse, A. Turrini, J. Int. Econ., 54, 97-117 (2001)

13. A. Bartel, F.Lichtenberg, Rev. Econ Stat., 69, 1-11 (1987)

14. A. Bartel, N. Sicheman, JPE, 107, 285-325 (1999)

15. A. Bernard, J. Eaton, B. Jensen, S. Kortum, AER, 94, 1265-1290 (2003)

16. S. Yeaple, J. Int. Econ., 65, 1-20 (2005)
17. E. Helpman, O. Itskhoki, S. Redding, Econometrica, 78, 1239-1283 (2010)

18. A. Filatov, Y. Sokolovsky, Proc. XIV April Int. Acad. Conf. on Econ. and Soc. Development, 1, 447460

19. L. Echazu, V. Lugovskyy (Working Paper, 2007).

20. A. Filatov, Y. Sokolovsky, Proc. XVI April Int. Acad. Conf. on Econ. and Soc. Development, 1, 548557 (2016)

21. A. Filatov, Y. Sokolovsky, Scientific Enquiry in the Contemporary World: Theor. Basics and Innov. Appr., 42-46 (2012) 